\title{
QUICK-RESPONSE PROTECTION SYSTEM AGAINST ELECTRIC SHOCK IN DISTRIBUTED GENERATION SYSTEMS
}

\section{Fedir SHKRABETS ${ }^{1}$, Sergej PLAKSIN ${ }^{2}$, Oleksandr OSTAPCHUK ${ }^{3}$, Valeriy KUZNETSOV ${ }^{4}$, Irina TYMCHENKO ${ }^{5}$, Antonina MUNTIAN ${ }^{6}$}

${ }^{1}$ Department of Power Engineering, Ukrainian State University of Chemical Technology, Gagarina 8, Dnipro, Ukraine, e-mail: shcrabetsf@nmu.org.ua

${ }^{2}$ Department of Control Systems, Institute of Transport Systems and Technologies of NAS of Ukraine, Pisarzhevsky str., 5, Dnipro, Ukraine, e-mail: svp@westa-inter.com

${ }^{3}$ Department of Renewable Energy, Igor Sikorsky Kyiv Polytechnic Institute, Peremohy ave., 37, Kyiv, Ukraine, 03056, e-mail: O.Ostapchuk@kpi.ua

${ }^{4}$ Electric Power Department, Railway Research Institute_4, Chlopickiego str., 50, Warsaw, Poland, e-mail: vkuznetsov@ikolej.pl

${ }^{5}$ Department of Control Systems, Institute of Transport Systems and Technologies of NAS of Ukraine, Pisarzhevsky str., 5, Dnipro, Ukraine, svp@westa-inter.com

${ }^{6}$ Foreign Language Department, Dnipro National University of Railway Transport, Lazariana 2, Dnipro, Ukraine, e-mail: muntonya@gmail.com

\begin{abstract}
The peculiarities of the operation of distributed generation systems lead to a change in the requirements for the reliability and safety of the power distribution systems in which they are integrated. To meet the increased requirements, continuous monitoring of the insulation parameters (active resistance and capacitance to ground) is often used with operating artificial non-industrial frequency operational signals introduced into the network. In the case of a sudden touch, such a system is not effective, since the response time of the protection device, which consists of the signal processing time and the operating of the actuator, is crucially important. For the used operational signals with a frequency of 100 and $200 \mathrm{~Hz}$, the processing time using the Fourier transform is $10 \mathrm{~ms}$ for $100 \mathrm{~Hz}$ and $5 \mathrm{~ms}$ for $200 \mathrm{~Hz}$. Considering that the response time of the fastest actuators (based on vacuum switches) is from 3 to $7 \mathrm{~ms}$, this is a rather significant period of time. To develop an effective resolver system, the capabilities of the Matlab environment were used to determine the most successful design of an analog prototype (Butterworth, Bessel, Chebyshev and Cauer of $6^{\text {th }}$ order) for an operational signal with a frequency of $200 \mathrm{~Hz}$. As a result, the processing time was established, which varies widely from 3.5 to $19 \mathrm{~ms}$. Taking into account the known indicators, namely the frequency and number of operational signals, a signal processing system was developed using the vector-matrix analysis method. As a result of modeling the processing characteristics of functional signals (at a sampling rate of $1 \mathrm{kHz}$ ), the system quick-response was $3 \mathrm{~ms}$, with the possibility of its further decrease as its productiveness increases.
\end{abstract}

Keywords: electric networks, protection, reliability, ground fault current, electrical safety, protection against electric shock, signal processing system, analog filtering.

\section{INTRODUCTION}

The sustainable development of power supply systems in recent years is associated with the close integration of renewable energy sources (RES) [1]. This tendency is especially active in distributed networks with a voltage of $6-10 \mathrm{kV}$, which have the greatest extent and length [2]. The reliability of power supply and safety of use are the main requirements for these networks. Fulfillment of these requirements is associated with a guaranteed supply of electricity to consumers, it leads to an increase in the quantitative indicators of such facilities and, accordingly, to the rise in the number of accidents. The most significant percentage of damages in such networks is revealed in singlephase earth faults (SEF) [3, 4]. This circumstance leads to the widespread use of an isolated neutral in them with protection against SEF, which influences on the signal $[5,6]$. When using such system, the emergency currents depend to a greater extent on the capacity of the network, and do not acquire large values. Based on the peculiarities of such networks, the power supply to consumers is not disturbed, and such regime can last from several minutes to a couple of hours, while the most dangerous consequences is the probability of a dangerous ground current occurring for a nearby person [7]. For this purpose, in certain countries with a high population density (Israel, Western Europe, etc.), protection acts on disconnection, which significantly reduces the reliability of such networks. 
To increase the reliability and safety of service, such networks are often equipped with systems for monitoring insulation parameters relative to earth (active resistance and capacitance to ground in operating mode) [8]. Such systems are called nextgeneration networks, or "smart networks". Equipping them with means of constant monitoring to assess the state allows to fix the degree of gradual degradation of insulation or to control the value of the network capacitance and the inductance of the arc suppression coil for subsequent tuning into the network resonance in emergency mode [9].

\section{FORMULATION OF THE PROBLEM}

The efficiency of determining the required parameters depends on the technical implementation of monitoring systems, which include the method of monitoring insulation with voltmeters; control of insulation resistance by a direct current of an external source; gate circuits, etc. [10]. However, the greatest efficiency in modern conditions was shown by the method of overlapping of artificial operational signals with one [11-15] or even several [16] frequencies.

Using one frequency is more accessible but does not allow controlling all the necessary parameters. When using two operational signals with a higher than industrial frequency, the system is more complicated, but it maintains the desired network parameters since the method is differential. The essence of the method is the use of the simultaneous overlapping of two operational sinusoidal signals on the electrical network, the frequencies of which are not equal to each other and differ from the industrial one (Fig. 1). The figure shows the situation when the signal source is connected to the neutral of the supply transformer $\mathrm{T}$, and the monitored parameters are taken at the outgoing connections from the TA and TV.

The information from the sensors is processed using microcontrollers MK. The choice of frequencies $f_{1}$ and $f_{2}$ of operational signals is determined from the condition that there are no harmonics of these frequencies in the voltage curve in normal mode (even and not multiples of three).

The choice of frequencies of operational signals is determined from the condition of aliases absence of these frequencies in the standard mode voltage curve (even and not multiples of three). Under such conditions, it is advisable to use an overlapping signal source based on standard circuits with frequency multipliers (two- and a fourfold increase in frequency). Insulation resistance values $R$, compensating device inductance $L_{p}$, and total capacity $C$ relative to the ground of the three phases of the entire network or the corresponding controlled section (connection) are calculated based on the measured parameters of the operational signals by the expressions

$$
\begin{array}{r}
R=U_{1} U_{2} \sqrt{\frac{\omega_{2}^{2}-\omega_{1}^{2}}{U_{2}^{2} I_{1}^{2} \omega_{2}^{2}-U_{1}^{2} I_{2}^{2} \omega_{1}^{2}}} \\
L_{p}=\frac{U_{1} U_{2}}{\omega_{1} \omega_{2}} \sqrt{\frac{\omega_{1}^{2}-\omega_{2}^{2}}{U_{1}^{2} I_{2}^{2}-U_{2}^{2} I_{1}^{2}}} \\
C=\frac{1}{U_{1} U_{2}} \sqrt{\frac{U_{2}^{2} I_{1}^{2}-U_{1}^{2} I_{2}^{2}}{\left(\omega_{1}^{2}-\omega_{2}^{2}\right)}}
\end{array}
$$

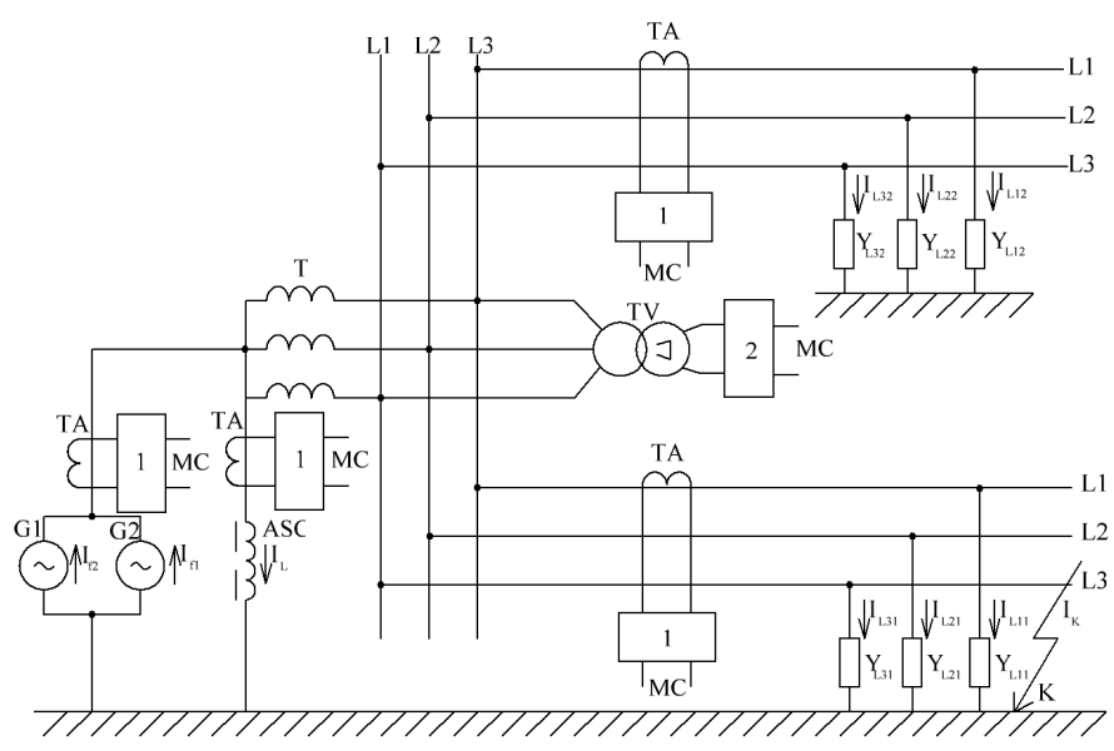

Fig. 1. Diagram explaining the method of continuous monitoring of the insulation parameters of the distribution network 1 - a device for isolating and processing of operating currents; 2 - a device for isolation and processing of operational voltages (G1, G2 - signal generator; TA, TV - current and voltage transformer; MC microcontroller; ASC - arc suppression coil) 
where $U, I, \omega-$ values of corresponding voltage, current and angular frequency of imposed operational sinusoidal signals with frequency $f_{1}$ and $f_{2}$

The use of the described method in real-time allows to determine the change in the monitored indicators and perform operational actions. A common disadvantage of such systems is their relatively low response time, which depends on the time required for extracting of the useful signal and operation of the actuator. When extracting signal, standard digital filtering systems are used, which advantages are the possibility of implementation on various computing devices: microcontrollers (MC), digital signal processor (DSP), a programmable logic device (PLD) etc. In these systems, the Fourier transform is most often used, in which the time delay depends on the period of the signal processing. In this case, the time for a signal with a frequency of $100 \mathrm{~Hz}$ is $10 \mathrm{~ms}$, and with $200 \mathrm{~Hz}$ is 5 $\mathrm{ms}$. When designing protection devices, it must be considered that their response time is the total response time of the signal processing system and the actuator. The response time of the actuator based on a modern vacuum circuit breaker is 3-7 ms [17]. Thus, the signal processing time is significantly longer than the response time of the actuator. Considering this circumstance and the proposition [18], which points that time is a significant indicator for ensuring human safety, it is necessary to develop data processing systems with a higher speed.

\section{SOLUTION METHODS AND ALGORITHMS}

When designing digital filtering systems, computer modeling tools are widely used, among which the MATLAB environment has become the main standard. In this software product, the design process is divided into specific stages. The first step is to determine the parameters of a digital filter based on an analog prototype. According to the recommendations in [19], the modeling of the most common analog lowpass filters (LPF) of the sixth order, namely: Butterworth, Bessel, Chebyshev, Cauer, was carried out. The modeling results are presented in Figures 2 - 6, where the $\mathrm{X}$-axis shows the frequency values in $\mathrm{Hz}$, and the $\mathrm{Y}$-axis shows the group delay in ms.

From the given dependencies, it can be concluded that the best quick-response of the filtering device for a signal with a frequency of the operational signal of $200 \mathrm{~Hz}$ varies in the range of 3.4-19 ms. Considering that these are the most favorable conditions, and for the frequency of 100 $\mathrm{Hz}$ the time interval is by 2 times longer, these results cannot be satisfactory already at the first design stage.

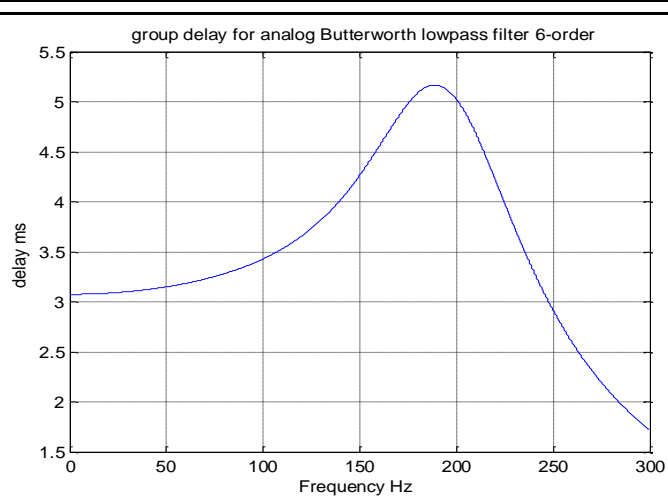

Fig. 2. GTD LPF with cutoff frequency $200 \mathrm{~Hz}$, Batterworth

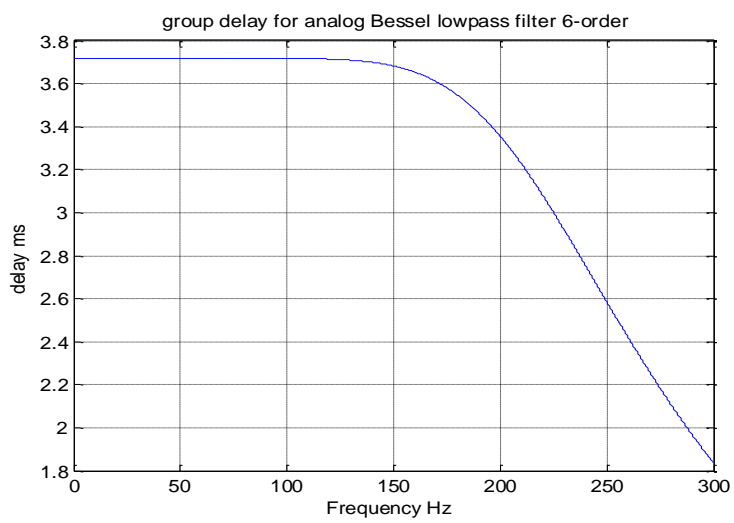

Fig. 3. GTD LPF with cutoff frequency $200 \mathrm{~Hz}$, Bessel.

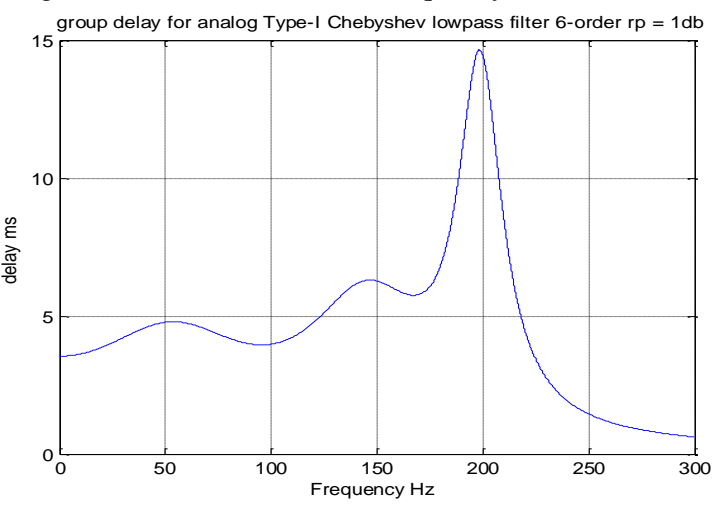

Fig. 4. GTD LPF with cutoff frequency $200 \mathrm{~Hz}$, Chebychev1.

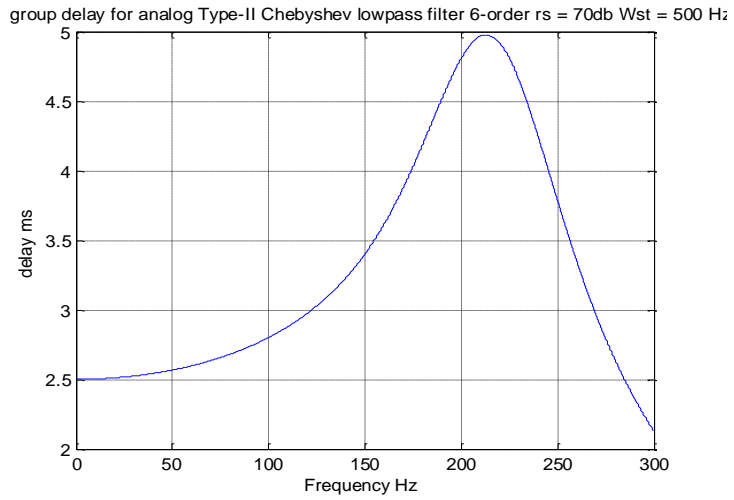

Fig. 5. GTD LPF with cutoff frequency $200 \mathrm{~Hz}$, Chebychev2. 


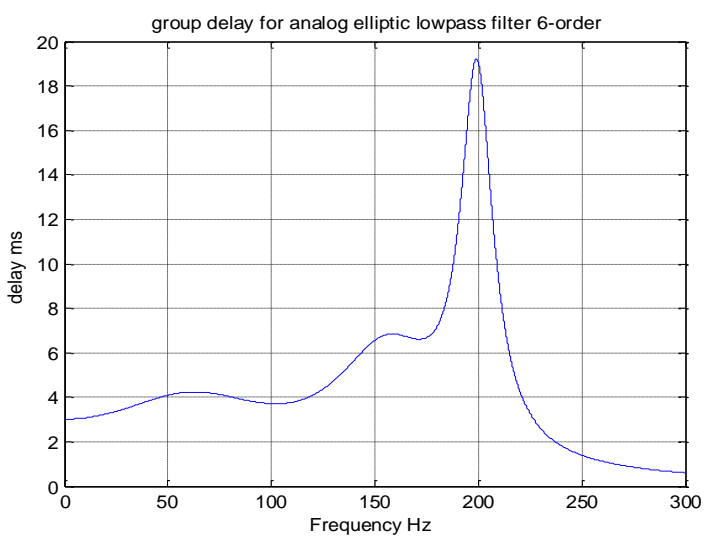

Fig. 6. GTD LPF with cutoff frequency $200 \mathrm{~Hz}$ Cauer (elliptic).

To develop filtering devices, it is necessary to take into account the peculiarities of the overlapping signal system and the fact that the parameters of the operational signals are known (periodicity, quantity, frequency, phase shift). In this case, the vector-matrix signal extraction method can be used, which is generally presented in [20]. In this method, in order to evaluate properly the amplitude quality of each of the two sinusoids, $2 * \mathrm{M}$ is necessary, where $\mathrm{M}=2$ (number of operational sinusoids) of the sampling rate periods. As a first step, the assumption is used that the operating signal model consists of the sum of cosine signals with amplitude $a_{k}$ and phase $\varphi_{k}$.

$x[n]=\sum_{k=1}^{2} a_{k} \cos \left(\omega_{k} n-\varphi_{k}\right)$; when $n=0,1,2, \ldots, N-1$

As already mentioned, the values of $\omega$ are known, and the number of samples is $N \geq 4$ and using the equation for the cosine of the sum of the angles, an expression is determined to represent the signal (2)

$x[n]=\sum_{\kappa=1}^{2} b_{k} \cos \left(\omega_{k} n\right)+d_{k} \sin \left(\omega_{k} n\right)$; when $n=0$, 1 ,

\section{2, 3}

where $-b_{k}=a_{k} \cos \left(\varphi_{k}\right)$ и $d_{k}=a_{k} \sin \left(\varphi_{k}\right)$.

Respectively, $a_{\mathrm{k}}=\sqrt{b_{k}^{2}+d_{k}^{2}}$ and $\varphi_{k}=\operatorname{arctg}\left(d_{k} / b_{k}\right)$. The equation (2) can be represented as a matrix $4 \times 1$ :

$$
\mathbf{X}=\left[\begin{array}{l}
x[0] \\
x[1] \\
x[2] \\
x[3]
\end{array}\right]
$$

or in vector form

$$
X=\sum_{k=1}^{2}\left(b_{k} c_{k}+d_{k} s_{k}\right)
$$

where $c_{k}$ and $s_{k}$ are vectors of cosines and sines of dimension $4 \times 1$, in the form

$$
c_{k=}\left[\begin{array}{l}
\cos \left(0 \omega_{k}\right) \\
\cos \left(\omega_{k}\right) \\
\cos \left(2 \omega_{k}\right) \\
\cos \left(3 \omega_{k}\right)
\end{array}\right], s_{k}=\left[\begin{array}{l}
\sin \left(0 \omega_{k}\right) \\
\sin \left(\omega_{k}\right) \\
\sin \left(2 \omega_{k}\right) \\
\sin \left(3 \omega_{k}\right)
\end{array}\right]
$$

As a result of the transformation $b_{k}$ and $d_{k}$, vector $\mathbf{f}(4 \times 1)$, vectors $c_{k}$ and $s_{k}$ are formed as a matrix $\mathbf{H}(4 \mathrm{x} 4)$

$$
\begin{gathered}
\mathbf{H}=\left[c_{1} s_{1} c_{2} s_{2}\right] \text { or } \\
{\left[\begin{array}{l}
\cos \left(0 \omega_{1}\right) \sin \left(0 \omega_{1}\right) \cos \left(0 \omega_{2}\right) \sin \left(0 \omega_{2}\right) \\
\cos \left(1 \omega_{1}\right) \sin \left(1 \omega_{1}\right) \cos \left(1 \omega_{2}\right) \sin \left(1 \omega_{2}\right) \\
\cos \left(2 \omega_{1}\right) \sin \left(2 \omega_{1}\right) \cos \left(2 \omega_{2}\right) \sin \left(2 \omega_{2}\right) \\
\cos \left(3 \omega_{1}\right) \sin \left(3 \omega_{1}\right) \cos \left(3 \omega_{2}\right) \sin \left(3 \omega_{2}\right)
\end{array}\right]}
\end{gathered}
$$

The last expression, which is used to determine the desired function $\mathbf{f}$, is obtained using the transposed matrix

$$
\mathrm{f}=\left(\mathrm{H}^{\mathrm{T}} \cdot \mathrm{H}\right)^{-1} \mathrm{H}^{\mathrm{T}} \mathrm{X}
$$

To determine the amplitudes of the first and second operational signals, the vector $\mathbf{f}$ is used

$$
a_{1}=\sqrt{\left(b_{1}^{2}+d_{1}^{2}\right)} \text { и } a_{2}=\sqrt{\left(b_{2}^{2}+d_{2}^{2}\right)}
$$

The functional diagram of the system for the given method is shown in Figure 7.

The effectiveness of the proposed method was tested in the MATLAB environment. Estimation of amplitudes of operational signals was performed in band with $200 \mathrm{~Hz}$ and sampling frequency of 1 $\mathrm{kHz}$. Figure 7 shows the dependences of the amplitude of the input and output signal. Input signal is in the form of a superposition of operational signals frequencies of 100 and $200 \mathrm{~Hz}$ (blue line) and estimation of amplitudes of an input signal with a frequency of $100 \mathrm{~Hz}$ (green line) and $200 \mathrm{~Hz}$ (red line).

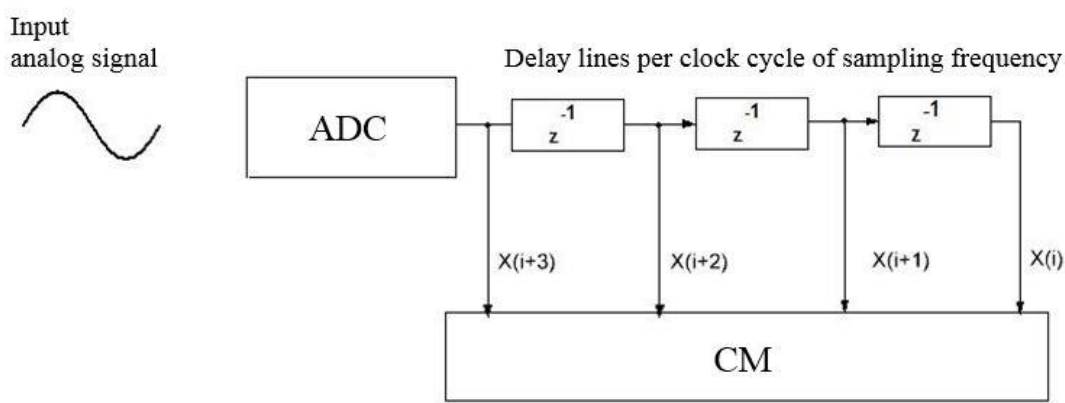

Fig. 7. Functional diagram of the proposed filtration system:

$\mathrm{ADC}$ - analogue-digital converter; $\mathrm{CM}$ - computing module; $\mathrm{Z}^{-1}$ - signal delay for one cycle of the sampling frequency 


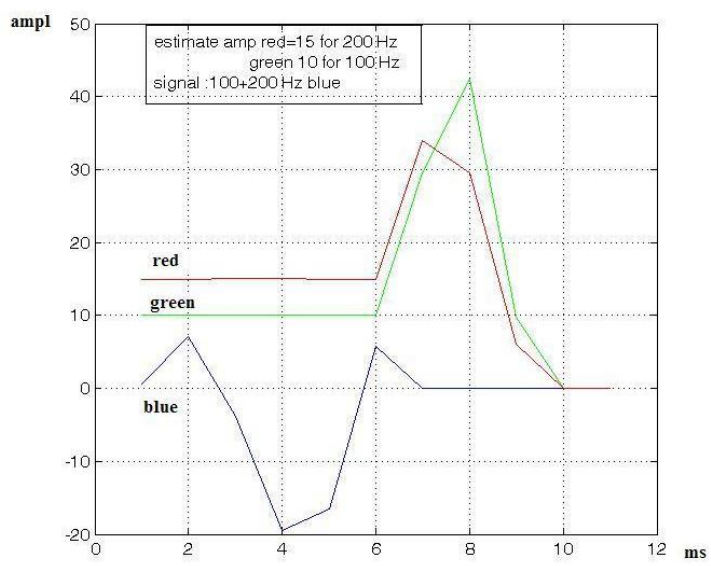

Fig. 7. Dependence of the amplitude of operational signals when simulating their rapid reduction to zero

According to the given algorithm, the estimation of amplitudes of operational signals in a band of $200 \mathrm{~Hz}$ and sampling frequency of $1 \mathrm{kHz}$ was modeled. In the input signal band, there is a superposition of the frequencies of the operational signals of 100 and $200 \mathrm{~Hz}$ (see Fig.7). Two separate signals of corresponding frequencies are received at the output. The processing speed depends on the sampling frequency and in this case, is equal to 3 ms. With a frequency of $10 \mathrm{kHz}$, the delay is 0.3 $\mathrm{ms}$. But it should be referring to an increase in the amount of computing, which requires the appropriate capabilities of the processor. Due to the fact that the issue of productivity today has no significant limitations, the processing delay can be considered insignificant compared to the delay of the analogy LPF if it is present at the ADC input.

Hardware implementation for testing the abovementioned algorithm is presented in Fig.8.

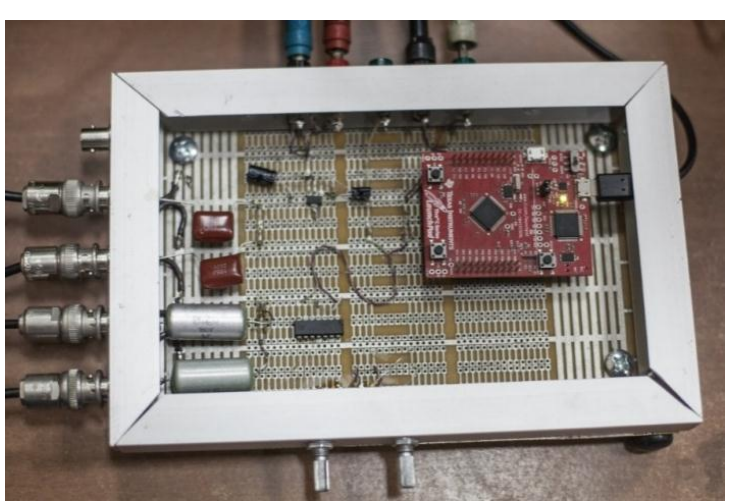

Fig. 8. General view of the filtering device based on the evaluation board EK-TM4C123GXL LaunchPad Evaluation Kit

The general scheme of the debug device of such systems is represented as structures in Fig. 9.

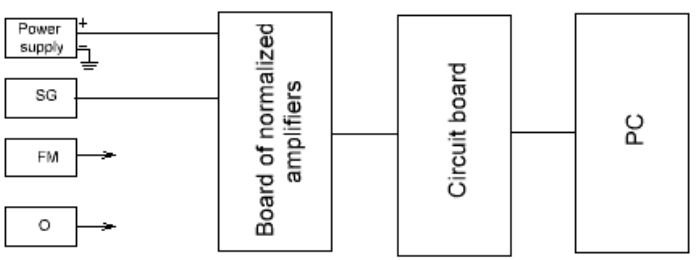

Fig. 9. General structure of the debug device: $S G$ - signal generator, FM - frequency meter, O - oscillograph, PC personal computer

The stand includes: power supply, signal generators of the corresponding frequency $Г 3-119$, frequency meter, oscillograph, board of normalizing amplifiers, evaluation board, and also the personal computer with the established special software. Verification of the proposed technical solutions was introduced on the basis of a specially built stand of simulation signals, the block diagram of which is shown in Fig. 10.

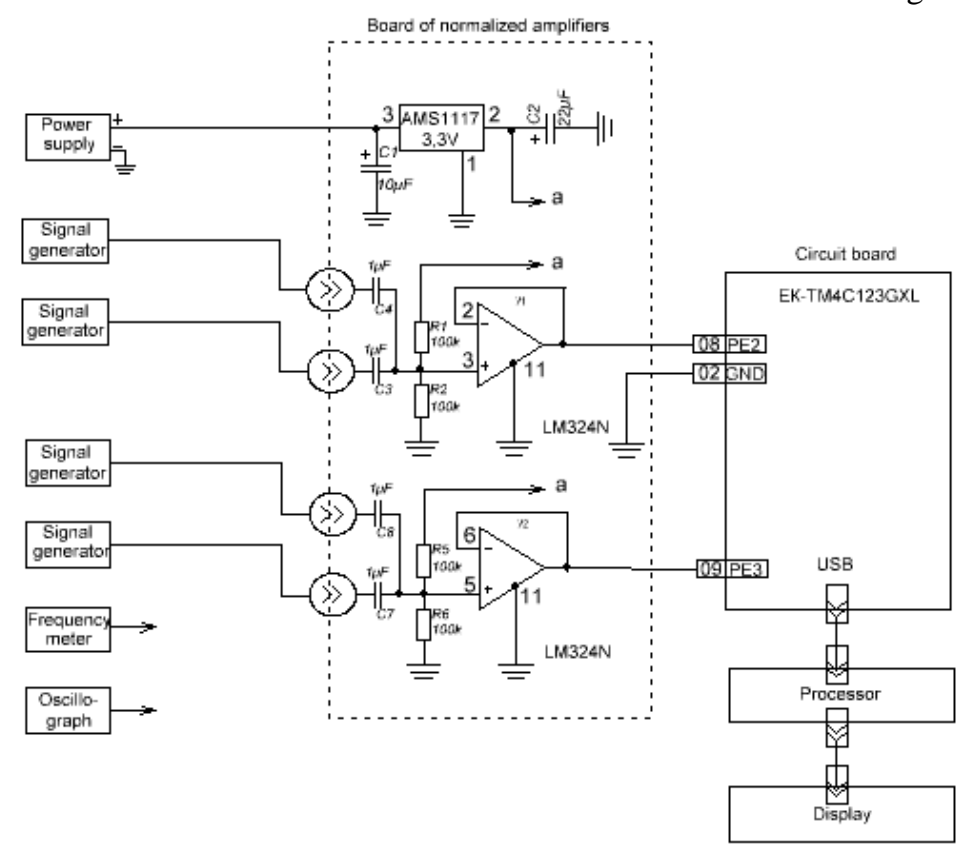

Fig. 10. Block diagram of the simulation stand of signals and debug the equipment 
The stand works as follows. Simulation signals (operational) are generated by four precision lowfrequency generators (Г3-119). The first pair of generators simulates functional voltage signals with frequencies of 100 and $200 \mathrm{~Hz}$. Their summation is carried out on the resistor R2. Then the total signal arrives to the direct input 3 of the operational amplifier LM324N with unity gain, in which the normalization of the total signal by amplitude is carried out and it binds by polarity to the ADC, which are installed in the microcontroller. The ADC parameters of the microcontroller are such that its operating voltage for the input signals varies in the range $0 \ldots+3.3 \mathrm{~V}$.

To use the dynamic range of this ADC in full (its bit grid of 12 bits), with zero total signal, the input of the ADC is supplied with a voltage of $+1.65 \mathrm{~V}$, which allows measuring an alternating signal with an amplitude of $1.65 \mathrm{~V}$ with a measuring step of about $0.8 \mathrm{mV}$.

The second pair of generators simulates the operating current signals with frequencies of 100 and $200 \mathrm{~Hz}$, which are synchronous with the operating voltage signals. For this pair of generators, the second operational amplifier works similarly to the first channel, but its output signal is sent to the second ADC of the microcontroller.

With the help of the developed program, the processing speed of the input signal with a frequency of $100 \mathrm{~Hz}$ and $200 \mathrm{~Hz}$ was checked to detect the time delay at a sampling frequency of 1 $\mathrm{kHz}$. For this purpose, a rapid voltage drop from the maximum value to zero was simulated for a time much lower than the sampling frequency. In fig. 7 the input signal decreases to 0 on the $7^{\text {th }}$ msec. The registration of the zero transition appears on the $10^{\text {th }}$ $\mathrm{ms}$, i.e. the delay in detecting the fact of reducing the voltage of the superposition of operational signals, as a sign of the network resistance violation, does not exceed $3 \mathrm{~ms}$. The offset between current and voltage signals can be set by the operating modes of the generators. Control of the frequency of signals and visual control of signals is carried out by means of the frequency meter 43-34 and the oscillograph C1-65A accordingly. The amplifiers are powered by the AMS1117 microstabilizer with a nominal voltage of $3.3 \mathrm{~V}$. In turn, the microstabilizer itself receives power from an external source with a voltage of 5-15 V. The software debug board is entirely standalone and connects to a PC via USB.

\section{RESULTS}

With the help of the developed program, the processing speed of the input signal with a frequency of $100 \mathrm{~Hz}$ and $200 \mathrm{~Hz}$ was checked to detect the time delay at a sampling frequency of 1 $\mathrm{kHz}$. For this purpose, a rapid voltage drop from the maximum value to zero was simulated for a time that is much shorter than the sampling period.
The indicator of reducing the voltage of the input signal to zero is the marker of the system's efficiency. In fig. 7 the input signal decreases to zero on the $7^{\text {th }} \mathrm{ms}$. The registration of the zero transition occurs on the $10^{\text {th }} \mathrm{ms}$, i.e. the delay in detecting the fact of reducing the voltage of the superposition of operational signals, as a sign of the network resistance violation, does not exceed $3 \mathrm{~ms}$. Thus, the processing speed at a sampling frequency of $1 \mathrm{kHz}$ is equal to $3 \mathrm{~ms}$. If the sampling rate is increased to $10 \mathrm{kHz}$, the delay is $0.3 \mathrm{~ms}$. It should be mentioned that the amount of computation that requires the appropriate capabilities of the processor increases. Since the issue of productivity today has no significant limitations, and the processing delay can be considered insignificant compared to the delay of any other LPF, in the case of its use at the input of the ADC.

\section{CONCLUSION}

In this work, establishing new patterns in the processing of operational signals in a limited time is accomplished. The dependences of the amplitude of the input and output signals in time are specified. An indicator of an accident is a decrease in the voltage of the superposition of operational signals as a sign of violation of the network resistance.

The practical value of the performed work is in developing of a methodology for determining the sampling rate. The obtained numerical values allow substantiating the cycle frequency of the microcontroller to ensure the required system speed. As a result of the implementation of the proposed technique, the speed of the operational signal processing system is increased and the operating time of the protection device in case of electric shock is reduced.

\section{ACKNOWLEDGMENTS}

The results were obtained within the project cofunded by the Polish National Agency for Academic Exchanges

(PPN/BUA/2019/1/00016/U/00001) and the Ministry of Education and Science of Ukraine.

\section{REFERENCES}

1. Renewable Energy in Poland. 2009;(November):20.

2. Ramesh MV, Mohan N, Devidas AR. Micro grid architecture for line fault detection and isolation. SMARTGREENS 2015 - 4th Int Conf Smart Cities Green ICT Syst Proc. 2015;250-5.

3. Hare J, Shi X, Gupta S, Bazzi A. Fault diagnostics in smart micro-grids: A survey. Renew Sustain Energy Rev. 2016;60:1114-24. http://dx.doi.org/10.1016/j.rser.2016.01.122.

4. Ostapchuk O, Kruczek W, Kuznetsov V, Kuznetsov VV, Tsyplenkov D. Analysis of the neutral grounding modes influence on the reliability characteristics of local systems with renewable energy sources. Diagnostyka. 2021;22(1):45-56. http://dx.doi.org/10.29354/diag/132834. 
5. Javadian SAM, Haghifam MR, Fotuhi Firoozabad M, Bathaee SMT. Analysis of protection system's risk in distribution networks with DG. Int J Electr Power Energy Syst. 2013;44(1):688-95. http://dx.doi.org/10.1016/j.ijepes.2012.08.034.

6. Conti S. Analysis of distribution network protection issues in presence of dispersed generation. Electr Power Syst Res. 2009;79(1):49-56.

7. Lopes JAP, Hatziargyriou N, Mutale J, Djapic P, Jenkins $\mathrm{N}$. Integrating distributed generation into electric power systems: A review of drivers, challenges and opportunities. Electr Power Syst Res. 2007;77(9):1189-203.

8. Van Vugt P, Bijman R, Timens RB, Leferink F. Impact of grounding and filtering on power insulation monitoring in insulated terrestrial power networks. IEEE Int. Symp. Electromagn Compat. 2013;472-7.

9. Jiang $\mathrm{H}, \mathrm{Xu} \mathrm{G}, \mathrm{Li} \mathrm{Q}$. Research on insulation state monitoring network technique for vessel electric network. ICEMS 2005 Proc Eighth Int Conf Electr Mach Syst. 2005;3:2250-3.

10. Olszowiec P. Insulation Measurement and Supervision in Live AC and DC Unearthed Systems. 2013.

11. Cong W, Pan Z, Zheng G, Jing H, Zhang F, Zhang Q. Study on single phase to ground fault site location method based on injection signal and GSM short message. IET Conf Publ. 2008;1(536 CP):365-9.

12. Li J, Liang J. 10kV straight line fault location based on Signal injection method. PEAM 2011 - Proc 2011 IEEE Power Eng Autom Conf. 2011;2:512-5.

13. He Z, Zhang J, Li WH, Lin X. Improved faultlocation system for railway distribution system using superimposed signal. IEEE Trans Power Deliv. 2010;25(3):1899-911.

14. Yi M, Huijiao L. An improved matrix algorithm for fault location in distribution network. Proc 2013 3rd Int Conf Intell Syst Des Eng Appl ISDEA 2013. 2013;289-93.

15. Bai Y, Cong W, Li J, Ding L, Lu Q, Yang N. Single phase to earth fault location method in distribution network based on signal injection principle. DRPT 2011 - 2011 4th Int Conf Electr Util Deregul Restruct Power Technol. 2011;(50807032):204-8.

16. Druml G. New method for the state evaluation of the zero-sequence system. 2005; (15th PSCC, Liege, 2226 August 2005):Session 39, Paper 2, Page 1-7.

17. Shkrabets F. Implementation of the insulation resistance control method for high-voltage grids of coal mines /F. Shkrabets, O. Ostapchuk//Power Engineering, Control and Information Technologies in Geotechnical Systems: Taylor \& Francis Group, London.

18. Vacuum Circuit Breakers 06C1-E-0004a http://www.fujielectric.co.jp

19. EN 50522: 2011 Groundingof power installations exceeding $1 \mathrm{kV}$ AC.

20. Giron-Sierra J.M. Digital Signal Processing with MATLAB Examples, Volume 1: Signals and Data, Filtering, Non-stationary Signals, Modulation Universidad Complutense de Madrid, Madrid, Spain, Springen, 2017. http://dx.doi.org/10.1007/978-98110-2534-1.

21. Estimation of the Amplitude and Phase of Sinusoids /Barry Van Veen// Access mode: https://allsignalprocessing.com/estimation-of-theamplitude-and-phase-of-sinusoids-in-noise
Received 2021-04-17 Accepted 2021-08-13 Available online 2021-08-17

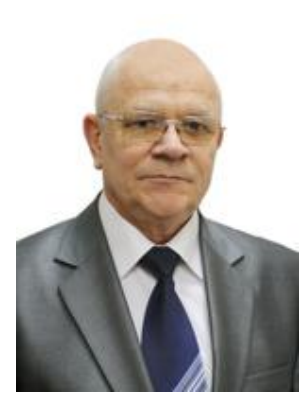

Fedir SHKRABETS

prof., dr. hab. Department of Power Engineering, Ukrainian State University of Chemical Technology, Ukraine.

Research interests:

Power supply systems of industrial enterprises, problems of integration of distributed generation sources into the power system, relay protection, electrical part of stations and substations.

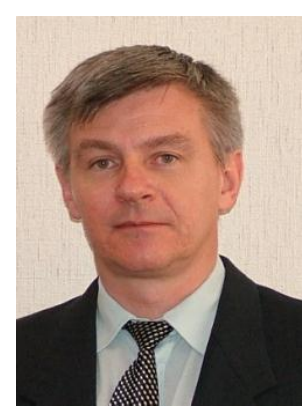

Sergej PLAKSIN

prof., dr.hab. Department of Control Systems, Institute of Transport Systems and Technologies, Ukraine Research interests: Power supply systems of industrial enterprises, signal processing, relay protection, intelligent power supply systems, renewable energy systems.

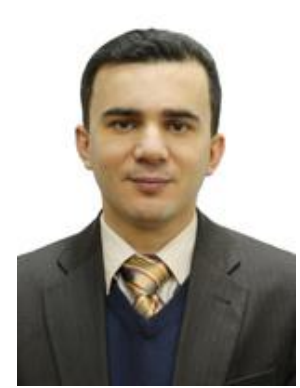

\section{Oleksandr OSTAPCHUK}

prof. (associate), dr.hab. Department of Renewable Energy, Igor Sikorsky Kyiv Polytechnic Institute. Research interests: Power supply systems of industrial enterprises, problems of integration of distributed generation sources into the power system, relay protection, electricity metering systems, intelligent power supply systems, electrical part of stations and substations.

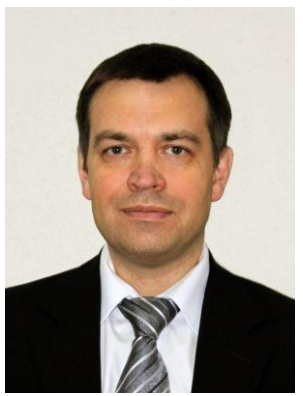

Valeriy KUZNETSOV prof., dr.hab. Electric Power Department, Railway Research Institute, Poland. Obtained dr.hab. degree in electric transport in 2012. His research interests concern: Energy saving methods, energy management, reliability of traction power supply systems.

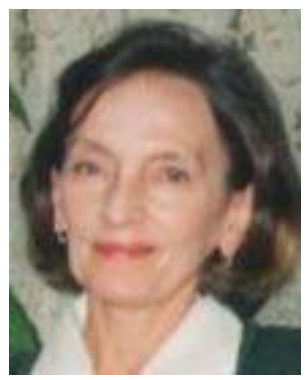

\section{Irina TIMCHENKO}

Department of Control Systems, Institute of Transport Systems and Technologies, Ukraine Research interests: Power supply systems of industrial enterprises, signal processing, relay protection, intelligent power supply systems, renewable energy systems. 